\title{
Psychosomatische Sprechstunde im Betrieb - Gestaltungsformen des Angebots
}

\section{Psychosomatic Consultation in the Workplace - Description and Modelling of a New Health-Related Service}

Autoren

Institute

\section{Preiser ${ }^{1}$, A. Wittich ${ }^{2}$, M. A. Rieger ${ }^{1}$}

${ }^{1}$ Institut für Arbeitsmedizin, Sozialmedizin und Versorgungsforschung, Universitätsklinikum Tübingen, Tübingen ${ }^{2}$ Supervisionsdienst am Klinikum, Klinik für Psychosomatische Medizin und Psychotherapie, Universitätsklinikum Freiburg, Freiburg

\section{Zusammenfassung}

$\nabla$

Ziel der Studie: In Deutschland nehmen Arbeitsunfähigkeitszeiten und Erwerbsunfähigkeitsrenten wegen psychischer Erkrankungen zu. Zugleich bestehen oft lange Wartezeiten in der ambulanten psychotherapeutischen Versorgung. Größere Betriebe reagieren hierauf mit dem Angebot einer „Psychosomatischen Sprechstunde im Betrieb“ (PSIB). Deren Ausgestaltung sollte explorativ beforscht werden.

Methodik: In einem qualitativen Ansatz (Einzelinterviews von innerbetrieblichen Akteuren und Fokusgruppen von Betriebsärzten; Inhaltsanalyse nach Mayring) wurde untersucht, wie sich derartige Angebote bedarfsgerecht ausgestalten lassen.

Ergebnisse: Die PSIB differiert hinsichtlich der innerbetrieblichen Information über das Angebot, der Zuweisungswege, des Orts der Intervention und ihres Umfangs. Ihre Ausgestaltung bewegt sich im Spektrum von „restriktiv“ bis „liberal“. Aus Sicht unterschiedlicher betrieblicher Akteure gibt es einen Bedarf für solche Angebote, sollte dem Betriebsarzt eine (zentrale) Rolle in dem Geschehen zukommen und hat sich die PSIB weitgehend bewährt. Zugleich beurteilen die Interviewten die damit verbundene Übernahme von Regelleistungen der Gesundheitsversorgung durch Arbeitgeber als zwiespältig.

Schlussfolgerung: Bei der Implementierung betriebsnah ausgerichteter Angebote der Krankenversorgung sollten spezifische Gestaltungsaspekte und Rahmenbedingungen berücksichtigt und die betrieblichen Akteure in den Prozess eingebunden werden.

\section{Einleitung}

$\nabla$

Auf psychische Erkrankungen entfielen im Jahr 2012 14,5\% der Arbeitsunfähigkeits(AU)-Tage und 4,5\% der AU-Fälle - bei durchschnittlich

\section{Abstract \\ $\nabla$}

Goal of the Study: In Germany, mental disorders have increasing importance for disability and early retirement. However, patients may have to wait several months before becoming an appointment with a psycho(somatic) therapist. Accordingly, several companies initiated a "psychosomatic consultation in the workplace" (PCIW). This concept has been explored.

Methods: Qualitative data analysis (expert interviews with stakeholders, focus group interviews with occupational health physicians; Mayring's content analysis) focussed on the question of how the concept of a PCIW can be tailored to meet the employees' needs.

Results: Concepts and implementation of PCIW differed with regard to the aspects dissemination of information about the consultation, gatekeeping, place of the consultation, and number of appointments with the psycho(somatic) therapist. The concepts of PCIW may be described as more or less "restrictive" or "liberal". The interviewees emphasised the need for PCIW and discussed the involvement of the occupational health physician within this approach. PCIW proved of value. Yet, the interviewees were ambivalent regarding the fact that companies offer and pay for treatment which should be provided within standard health care.

Conclusion: Shaping company-based elements of standard health care should respect settingspecific needs and involve in-company stakeholders into the process.

33,2 Krankheitstagen pro Fall (DAK-Versicherte [1]). Zugleich stellen sie aktuell mit $40 \%$ die häufigste Ursache für vorzeitigen Renten-Neuzugang wegen verminderter Erwerbsfähigkeit dar [2]. 
Beide Aspekte sind für Betriebe und Beschäftigte gleichermaßen bedeutsam. Entsprechend widmen sich betriebliche Akteure zunehmend der Primär- und Sekundärprävention psychischer Erkrankungen. Ansatzpunkte sind zum einen die betriebliche Sozialberatung bzw. psychosoziale Beratung [3] einschließlich der betrieblichen Suchthilfe [4] sowie Supervision und Coaching [5] - jeweils in Abhängigkeit von Betriebsgröße und Branche. Zum anderen werden Arbeitsbedingungen so gestaltet, dass psychische Fehlbelastungen möglichst vermieden werden - häufig unter Federführung der Betriebsärzte und ggf. unter Einbindung arbeitspsychologischer Expertise [6]. Dabei ist der betriebliche Gesundheitsschutz in großen Betrieben oft deutlich besser ausgestaltet als in kleinen und mittleren Unternehmen.

Bei drohender oder bestehender psychischer bzw. psychosomatischer Erkrankung, d.h. bei Sekundärprävention/Therapie, verweisen Betriebsärzte Beschäftigte - häufig vermittelt über den Hausarzt - auf die Regelversorgung. Allerdings bestehen hier lange Wartezeiten für ein Erstgespräch (aktuell: bis zu 2,2 Monate) bzw. den Beginn einer Psychotherapie (aktuell: bis zu 4,8 Monate) [7], Zahlen nach: [8].

Vor diesem Hintergrund wurden von Betriebsärztinnen und Betriebsärzten in einigen Unternehmen sogenannte „Psychosomatische Sprechstunden im Betrieb“ initiiert und in Zusammenarbeit mit den kooperierenden Fachärzten für Psychosomatik bzw. Psychiatrie oder psychologischen Psychotherapeuten ausgestaltet. Die Bezeichnung „Psychosomatische Sprechstunde im Betrieb“ (PSIB) ist hierbei der bisher einzigen Publikation eines entsprechenden Programms entlehnt [9] und wird nachfolgend als Überbegriff für Angebote in verschiedenen Firmen verwendet, die dort jeweils unterschiedlich benannt und ausgestaltet sind. Bei allen Formen wird den Beschäftigten bei psychosomatischen Beschwerden oder drohender bzw. bestehender psych(osomat)ischer Erkrankung ermöglicht, auf Kosten des Betriebes Zugang zu einem psychosomatisch spezialisierten Arzt oder psychologischen Psychotherapeuten zu erhalten. Analog zum Modell der Konsiliarpsychosomatik in der stationären Versorgung [10] findet - bei Einwilligung des Beschäftigten - auch im Modell der PSIB teilweise ein fachlicher Austausch zwischen den betreuenden Betriebsärzten und dem psychosomatischen Kollegen statt. Im Rahmen der bislang auf wenige Betriebe beschränkten PSIB erfolgen somit Angebote aus der kurativen Versorgung im betrieblichen Setting und ergänzen die betriebsärztliche Betreuung.

Verschiedene Autoren haben Vorschläge zur Entwicklung und Evaluation neuer Versorgungsangebote gemacht z.B. [11]. Im Hinblick auf die PSIB gilt es, deren Ausgestaltung zu beschreiben, um einen Beitrag zur Modellbildung bzw. ggf. Modifikation erster Angebote auf dem Boden der nachträglichen Theoriebildung zu leisten. Dies ist Ziel der vorliegenden Studie.

\section{Methode}

$\nabla$

Da zum Zeitpunkt der Studie jenseits einer ersten Darstellung zur PSIB [9] keine empirischen Daten vorlagen, wurde ein exploratives Forschungsdesign unter Verwendung qualitativer Methoden der empirischen Sozialforschung gewählt und Einzelinterviews sowie Fokusgruppendiskussionen mit „Experten“ durchgeführt. Fokusgruppen (FG) sind als Forschungsmethodik v.a. zur Datenerfassung in beruflichen Gruppen etabliert. Die Gruppendiskussion regt die Teilnehmenden an, sich vertieft mit Themen auseinanderzusetzen [12]. Der Expertenbegriff folgt
Meuser et al. [13] und fokussiert die Kombination aus spezifischem Wissen und Einbindung in Problemlösungsprozesse. Als Experten wurden neben Betriebsärzten (in Fokusgruppen) Vertreter innerbetrieblicher Beratungsdienste, Personalabteilungen, Betriebskrankenkassen und Personalvertretungen (in Einzelinterviews (EI)) angesprochen.

\section{Studienpopulation}

Planungsgemäß wurden $2 \mathrm{FG}$ realisiert: in einer wurden Betriebsärzte mit PSIB-Erfahrung, in der anderen Betriebsärzte ohne entsprechenden Hintergrund interviewt. Auch bei den EIs wurde eine strukturelle Variation [14] durch die Differenzierung „mit PSIB-Erfahrung“ - „ohne PSIB-Erfahrung“ sowie die Kategorie „großes Unternehmen“ - „kleines/mittleres Unternehmen" und die Abbildung verschiedener Berufsgruppen erreicht. Experten ohne Erfahrung mit dem Angebot wurden in die Studie eingeschlossen, da die Ausgangsproblematik der psych(osomat) ischen Erkrankungen angebotsunabhängig ist. Zudem wurden so potenziell betroffene Betriebe inkludiert.

Das Studiensample ist in $\bullet$ Tab. 1 dargestellt. Die PSIB-erfahrenen Betriebsärzte waren aufgrund der begrenzten Anzahl von Unternehmen, die zum Zeitpunkt der Studie eine PSIB in Südwestdeutschland anboten, leicht zu identifizieren: 5 der 7 in Frage kommenden Betriebsärzte nahmen an der FG teil, einer an einem EI, einer sagte aus Zeitgründen ab. Die Betriebsärzte ohne PSIB-Erfahrung wurden über die (Lehr-)Kooperationen des Instituts für Arbeitsmedizin, Sozialmedizin und Versorgungsforschung Tübingen rekrutiert (Teilnahmequote Fokusgruppe 5/6; 1 Absage aus Zeitgründen). Die weiteren betrieblichen Ansprechpartner für die EI wurden mithilfe der interviewten Betriebsärzte gewonnen (Teilnahmequote 8/8).

\section{Durchführung}

Die FGs wurden im Herbst 2011 durchgeführt (Dauer 94 und $102 \mathrm{~min}$ ). Die Els wurden mit einer Ausnahme telefonisch geführt (Dauer 19-34, im Mittel $26 \mathrm{~min}$ ). Alle Interviews wurden digital aufgezeichnet, die FG zusätzlich videodokumentiert (Löschung nach Zuordnung der Sprecher zu den Interviewpassagen). Die Interviews der Experten ohne PSIB-Erfahrung (EI und FG) erfolgten zeitlich nach der FG der Betriebsärzte mit PSIBErfahrung. Aus letzterem wurden als Prototypen 2 kontrastierte PSIB-Beispiele abgeleitet ( $\bullet$ Tab. 3), den Experten ohne Erfahrung vorgestellt und ihre Haltungen dazu erfragt. Die Prototypen dienten somit als in Fokusgruppendiskussionen üblicher Diskussionsimpuls [12].

\section{Auswertung}

Die Daten wurden transkribiert, pseudonymisiert und im Anschluss zum Zwecke der kommunikativen Validierung von 3 unabhängigen (neutralen) Personen ausgewertet. Die Auswertung erfolgte deduktiv aus bereits vorhandenem Wissen und von den Leitfragen ausgehend sowie induktiv - methodisch mittels zusammenfassender Inhaltsanalyse nach Mayring [15]. Hierbei wurde das in einem mehrstufigen Prozess entwickelte Kategoriensystem in der Software MAXQDA erstellt, für alle Interviews verwendet und beim Aufkommen neuer Aspekte erweitert. Eine inhaltliche Validierung erfolgte durch einen Workshop, zu dem alle Interviewpartner sowie weitere Vertreter derselben Berufsgruppen, Vertreter von Arbeitgeberverbänden und Gewerkschaften und des zuständigen Landesministeriums eingeladen waren. An diesem Workshop nahmen 26 Personen teil (Februar 2012). 
Tab. 1 Zusammensetzung der Studienpopulation (Kursiv: Kürzel der Interviews).

\begin{tabular}{|c|c|c|}
\hline & Erfahrung mit Angebot & Keine Erfahrung mit Angebot \\
\hline Betriebsärzte & $\begin{array}{l}\text { - } 5 \text { Betriebsärzte aus verschiedenen großen Unter- } \\
\text { nehmen der Metall- und Elektro- bzw. } \\
\text { Automobilindustrie (ca. } 2500 \text { bis>15000 Beschäf- } \\
\text { tigte am jeweiligen Standort) (FG_AMM-01)* } \\
\text { - } 1 \text { Metall- und Elektroindustrie (ca } 10500 \\
\text { Beschäftigte) (EM-02) }\end{array}$ & $\begin{array}{l}\text { - } 3 \text { freiberufliche Betriebsärzte, betreuen mehrere Unternehmen } \\
\text { (jeweils } \leq \text { ca. } 500-1000 \text { Beschäftigte) } \\
\text { - } 1 \text { Betriebarzt, überbetrieblicher Dienst, betreut ein Unternehmen der } \\
\text { Medizintechnik (ca. } 3000 \text { Beschäftigte) } \\
\text { - } 1 \text { Betriebsarzt, überbetrieblicher Dienst, betreut mehrere Unternehmen } \\
\text { (jeweils } \leq 1000 \text { Beschäftigte) (FG_AMO-01) }\end{array}$ \\
\hline Personalvertretung & - & $\begin{array}{l}1 \text { Krankenhaus der Maximalversorgung } \\
\text { (ca. } 9.000 \text { Beschäftigte) (EO-03) }\end{array}$ \\
\hline $\begin{array}{l}\text { Betriebskranken- } \\
\text { kasse }\end{array}$ & - & 1 Medizintechnik (ca. 3200 Beschäftigte) (EO-02) \\
\hline $\begin{array}{l}\text { Betriebliche Sozialbe- } \\
\text { ratung/Psychosoziale } \\
\text { Beratung }\end{array}$ & $\begin{array}{l}\text { - } 1 \text { Metall- und Elektroindustrie (ca } 10500 \\
\text { Beschäftigte) (EM-03), } \\
\text { - } 1 \text { Automobilindustrie, (> } 15000 \text { Beschäftigte) } \\
\text { (EM-01) }\end{array}$ & $\begin{array}{l}1 \text { tätig für einen großen überbetrieblichen arbeitsmedizinischen Dienst, } \\
\text { d. h. viele auch kleine und mittelgroße Betriebe (EO-05) }\end{array}$ \\
\hline Personalabteilung & - & $\begin{array}{l}2 \text { Metall- und Elektroindustrie (ca. } 3.000 \text { und ca. } 9000 \text { Beschäftigte) } \\
\text { (EO-01, EO-04) }\end{array}$ \\
\hline
\end{tabular}

Tab. 2 Gestaltungsformen der Psychosomatischen Sprechstunde im Betrieb - Kategorien und Zitatbeispiele.

\begin{tabular}{|c|c|}
\hline Hauptkategorie & Unterkategorie \\
\hline \multirow[t]{2}{*}{$\begin{array}{l}\text { „Innerbetriebliche } \\
\text { Information über die } \\
\text { Psychosomatische } \\
\text { Sprechstunde“ }\end{array}$} & $\begin{array}{l}\text { Zitat 1: Breite } \\
\text { Information }\end{array}$ \\
\hline & $\begin{array}{l}\text { Zitat 2: Begrenzte } \\
\text { Information }\end{array}$ \\
\hline \multirow[t]{2}{*}{$\begin{array}{l}\text { „Zugang bzw. } \\
\text { Zuweisung zur } \\
\text { Psychosomatischen } \\
\text { Sprechstunde“ }\end{array}$} & $\begin{array}{l}\text { Zitat 3: Breiter } \\
\text { Zugang }\end{array}$ \\
\hline & $\begin{array}{l}\text { Zitat 4: Begrenzter } \\
\text { Zugang/gezielte } \\
\text { Zuweisung }\end{array}$ \\
\hline \multirow[t]{2}{*}{$\begin{array}{l}\text { "Ort der Psychoso- } \\
\text { matischen Sprech- } \\
\text { stunde“" }\end{array}$} & $\begin{array}{l}\text { Zitat 5: Betriebs- } \\
\text { eigene Räumlich- } \\
\text { keiten }\end{array}$ \\
\hline & $\begin{array}{l}\text { Zitat 6: Räumlich- } \\
\text { keiten des externen } \\
\text { Konsiliars }\end{array}$ \\
\hline $\begin{array}{l}\text { „Umfang der } \\
\text { Behandlung“ }\end{array}$ & Zitat 7: Ein Termin \\
\hline
\end{tabular}

\section{Zitatbeispiel}

„(...) das war ganz wichtig (...), dass wirklich von der Unternehmungsleitung und vom Betriebsrat das System mitgetragen wird und in die Landschaft getragen wird, also klassisch wäre da eine Betriebsversammlung, wo beide Seiten das Thema in die Mannschaft tragen und sagen, „das ist was Gutes für Sie, das ist, kann auch hilfreich sein und wir unterstützen das von beiden Seiten.“ (FG_AMO-01, 132) „... Wir wollen das nämlich auch nicht mit der Gießkanne ausgießen.“ (EM-02, 51)

„Aber in den [kleineren] Betrieben sind die [Betriebsräte] in der Regel nicht so gut qualifiziert, dass sie das zusortieren können. Also insofern würde ich eher dazu tendieren, sollte der Betriebsarzt auf jeden Fall mit einbezogen werden, ob er da alleiniger Zuweiser ist, Fragezeichen, aber er muss davon in Kenntnis gesetzt werden." (FG_AMO-01, 47)

„Grundsätzlich finde ich die Idee nicht schlecht, sozusagen den Betriebsarzt da zum Dreh- und Angelpunkt dieser Themen zu machen. Der Betriebsarzt ist sicherlich schon jemand, der auch einen besseren Zugang nochmal hat, weil natürlich klar ist, dass alles vertraulich bei ihm bleibt.“ (EO-04, 51) „Auch dass es im Betrieb stattfindet wird eher positiv gesehen, es ist in der Arbeitszeit, was will man mehr, es gibt keine Anfahrtswege, also die Schwelle dort teilzunehmen ist extrem gering (...).“ (FG_AMM-01, 48)

„Generell würde ich aber aufgrund der Vertraulichkeiten, auch aufgrund von Berührungspunkten... eher einen externen Ort vorziehen wollen." (EO-01, 61)

„Was für mich ganz gut ist, ist dass ein Externer, der keinen Bezug zu dem Unternehmen hat und auch keinen Bezug zu der Umgebung, in der derjenige [Mitarbeiter] arbeitet, der sehr geschult ist von seiner Objektivität her, den Fall aus seiner Brille anschaut und dann zum einen dem Mitarbeiter Hilfestellung gibt, wie kann es für ihn weitergehen, (...) dass er [der Mitarbeiter] erkennt, es ist nicht nur die Umwelt, die mit ihm umgeht, sondern er ist auch ein Akteur in der Umwelt und kann sich entsprechend verändern, das sind dann so Fälle, wo oft der einmalige Termin dann auch reicht bei der Sprechstunde, um tatsächlich eine Veränderung zu bewirken.“ (FG_AMM-01, 30)

Zitat 8: Mehrere $\quad$ „(...) wir haben mit dem einen Partner einen Deal, dass man maximal 5 Sitzungen bei dem absolvieren Termine $\quad$ kann und diese 5 Sitzungen werden in den seltensten Fällen in Anspruch genommen. Nicht, weil es dem Mitarbeiter nicht weiterhilft, sondern weil er nach 2, 3 Sitzungen im Endeffekt sein Problem erkannt hat und auch Anleitung bekommen hat, wie er es umsetzen kann." (EM-03, 82)

„Bewertung und Z Zitat 9: Negative Reflexion“ Bewertung „Die Psychosomatische Sprechstunde wird teilweise auch verwendet, um etwas in Anspruch zu nehmen, was es schon gibt. Das heißt, wir haben hier eine Doppelstruktur. (...) Das ist für den Mitarbeiter egal, Hauptsache er bekommt einen Termin. Allerdings ist der dann [in der Psychosomatischen Sprechstunde] zeitlich meistens länger entfernt, als der nächstmögliche Termin [in einer innerbetrieblichen Beratungsstelle]. [Durch die Psychosomatische Sprechstunde] wird dann einfach eine vorhandene Struktur weniger genutzt und dafür eine andere in Anspruch genommen." (EM-01, 103)

Zitat 10: Positive Wenn Sie fragen, „Was könnte man verbessern?“, dann denke ich, wir haben eigentlich mit dieser Bewertung Psychosomatischen Sprechstunde, wie ich glaube, ein gutes Angebot.“ (FG_AMM-01, 68)

EM - Einzelinterview Experte mit Erfahrung mit Psychosomatischer Sprechstunde (PS), E0 - Einzelinterview Experte ohne Erfahrung mit PS, FG_AMM: Fokusgruppe Betriebsärzte mit Erfahrung mit PS, FG_AM0 Fokusgruppe Betriebsärzte ohne Erfahrung mit PS; Arabische Ziffern kennzeichnen die Pseudonymisierungsziffern der Interviewpartner sowie den Abschnitt in der MAXQDA-Datei 
Tab. 3 Ausprägungen der Gestaltungsaspekte der „Psychosomatischen Sprechstunde im Betrieb“.

\begin{tabular}{lll}
$\begin{array}{l}\text { Gestaltungsaspekt } \\
\text { Information }\end{array}$ & $\begin{array}{l}\text { „Restriktive“ Ausprägung } \\
\text { Selektive Information über das Angebot in der be- } \\
\text { triebsärztlichen Sprechstunde } \\
\text { Betriebsarzt als alleiniger „Zuweiser“ }\end{array}$ & $\begin{array}{l}\text { „Liberale“ Ausprägung } \\
\text { Breite Information über das Angebot im Betrieb }\end{array}$ \\
\hline $\begin{array}{l}\text { Zugang/Zuweiser } \\
\text { Ort }\end{array}$ & $\begin{array}{l}\text { Betriebsinterne Räumlichkeiten (i.d. R. Räumlichkei- } \\
\text { ten des betriebsärztlichen Dienstes) } \\
\text { ein Termin }\end{array}$ & $\begin{array}{l}\text { Heterogene „Zuweiser“, Einbindung des Betriebsarztes wäh- } \\
\text { rend des Prozesses }\end{array}$ \\
\hline $\begin{array}{l}\text { Umfäumlichkeiten des externen Konsiliar } \\
\text { Kosten des Arbeitgebers }\end{array}$ & & bis zu 5 Termine \\
\hline
\end{tabular}

\section{Finanzierung und ethische Aspekte}

Die Studie wurde gefördert durch das Ministerium für Arbeit und Sozialordnung, Familie, Frauen und Senioren Baden-Württemberg. Ein positives Votum der zuständigen Ethikkommission lag vor. Alle Teilnehmenden wurden schriftlich aufgeklärt und gaben ihr Einverständnis.

\section{Ergebnisse \\ $\nabla$}

Dieser Artikel greift aus den Studienergebnissen diejenigen zu möglichen Gestaltungsformen der PSIB auf. Nachfolgend werden die Themen „Innerbetriebliche Information über die Sprechstunde“, „Zugang bzw. Zuweisung“, „Ort der Sprechstunde“ und „Umfang der Behandlung“, sowie allgemein die „Bewertung und Reflexion des Angebotes“ dargestellt. Zugunsten einer möglichst unmittelbaren Wiedergabe der Interviewaussagen erfolgt die Darstellung im Präsens, unter Verzicht auf den Konjunktiv und ohne inhaltliche Zusätze der Autorinnen. Die jeweiligen Zitatpassagen finden sich unter der entsprechenden Nummerierung in 0 Tab. 2.

Die Ergebnisse werden nicht nach „mit Erfahrung“ - „ohne Erfahrung", sondern thematisch geordnet, da Themen und deren Dimensionen in beiden Gruppen ähnlich zur Sprache kamen.

\section{Kategorie „Innerbetriebliche Information über die Psychosomatische Sprechstunde"}

Ein Teil der Befragten spricht sich dabei dafür aus, alle Beschäftigten über Existenz und Zweck der Psychosomatischen Sprechstunde zu unterrichten, etwa in Betriebsversammlungen, wo Unternehmensleitung und Arbeitnehmervertreter gemeinsam informieren können (Zitat 1). Andere Befragte bevorzugen, dass Betriebsärzte oder andere Zuweisungsbefugte wie Mitarbeiter innerbetrieblicher Beratungsdienste einzelne Personen gezielt im persönlichen Gespräch informieren, um die differentielle Indikation sicherzustellen (Zitat 2, vgl. auch nächster Abschnitt).

\section{Kategorie „Zugang bzw. Zuweisung zur \\ Psychosomatischen Sprechstunde"}

Ein Teil der Befragten präferiert einen breiten Zugang zur PSIB, bei dem neben Betriebsärzten andere Akteure, wie Betriebsräte oder Mitarbeitende der Personalabteilung Beschäftigte der Sprechstunde zuweisen bzw. auf diese verweisen. Dies wird besonders für Unternehmen vorgeschlagen, in denen der Betriebsarzt nur selten vor Ort ist. Betriebsärzte selbst plädieren dafür, auch bei diesem breiten Zugang die Einbeziehung des Betriebsarztes zu gewährleisten (Zitat 3). Manche Befragte sprechen sich für einen begrenzten Zugang zur PSIB aus, bei dem allein der Betriebsarzt Beschäftigte zuweisen kann (Zitat 4).
Kategorie „Ort der Psychosomatischen Sprechstunde“

Der Ort der Psychosomatischen Sprechstunde ist unter mehreren Aspekten relevant. Wo die Sprechstunde in betriebseigenen Räumlichkeiten stattfindet, wird sie als regelmäßiger Blocktermin durchgeführt. Für betriebseigene Räumlichkeiten spricht aus der Sicht der Befragten, dass die Sprechstunde innerhalb der Arbeitszeit und niederschwellig stattfinden kann (Zitat 5). Befürchtungen hinsichtlich der Sicherstellung von Anonymität wird in der Praxis begegnet, indem betriebsärztliche Räumlichkeiten genutzt werden, sodass für Dritte nicht nachvollziehbar ist, wer die betriebsärztliche bzw. die Psychosomatische Sprechstunde nutzt.

Andere Befragte bevorzugen die Räumlichkeiten des externen Konsiliars, weil dadurch Termine individuell vereinbart werden können, wenngleich sich Wegstrecken negativ auf die Inanspruchnahme auswirken könnten. Zugleich werden die Reflexion außerhalb des betrieblichen Settings und der anonyme Zugang (Zitat 6) für förderlich gehalten.

In der Praxis finden sich beide Formen und werden von den Beteiligten als stimmig erachtet.

\section{Kategorie „Umfang der Behandlung“}

Die Angebote unterscheiden sich auch hinsichtlich des Umfangs der (betrieblich finanzierten) Behandlungszeit. Ein Teil der Betriebe übernimmt die Kosten für einen Termin, was einige Befragte als ausreichend und wirkungsvoll erachten (Zitat 7). Sie sehen es nicht als Aufgabe von Betrieben, Kassenleistungen zu übernehmen.

Andere Befragte bevorzugen ein Modell, in dem der Betrieb bis zu 5 Termine finanziert. Dies ermöglicht eine vertiefte Auseinandersetzung mit der Problematik und kann die Wirkung der Sprechstunde positiv beeinflussen. Manche berichten, dass auch dort, wo 5 Gesprächstermine möglich sind, diese nicht immer ausgeschöpft werden (Zitat 8).

\section{Kategorie „Bewertung und Reflexion der Psychosomatischen Sprechstunde“}

Kritisch wird die PSIB dann beurteilt, wenn sie als Doppelstruktur wahrgenommen wird, bei der der Betriebsarzt nicht eingebunden ist und der externe Kooperationspartner als Ersatz für betriebsärztliche Angebote wahrgenommen wird (Zitat 9). Insgesamt werden in den Interviews eine hohe Zufriedenheit bei den Experten „mit Erfahrung“ und eine hohes Interesse seitens der Experten ohne Erfahrung mit der PSIB deutlich (Zitat 10).

\section{Zusammenfassende Darstellung der Ergebnisse}

Hinsichtlich der Gestaltung der Psychosomatischen Sprechstunde befassten sich die Interviewten mit den Themen Information, Zugang, Ort und Umfang der konsiliarischen Behandlung. Aus 
den bereits etablierten Angeboten und den geäußerten Präferenzen und deren Begründungen lassen sich verschiedene Gestaltungsaspekte herausarbeiten ( $\bullet$ Tab. 3).

Modellhaft kann die PSIB eher „restriktiv“ gestaltet werden, indem es allein dem Betriebsarzt vorbehalten ist, ausgewählte Beschäftigte darüber zu informieren und der Sprechstunde zuzuweisen, diese vor Ort stattfindet und vom Arbeitgeber nur im Umfang von einem Termin finanziert wird. Demgegenüber wird in einem durchgehend „liberalen“ Modell das Angebot breit an alle Beschäftigte kommuniziert, es gibt verschiedene Zuweiser, die Sprechstunde findet betriebsextern in Räumen des Konsiliars statt und wird im Umfang von bis zu 5 Terminen vom Arbeitgeber finanziert. Zwischen diesen Polen sind verschiedene andere Modelle denkbar - und finden sich auch in der Praxis ( $\bullet$ Tab. 4). Die befragten Betriebsärzte wünschen unabhängig von der Ausprägung der einzelnen Gestaltungsaspekte eine starke Einbindung des Betriebsarztes innerhalb der PSIB. Bei den anderen befragten Berufsgruppen sind die Präferenzen heterogen.

\section{Diskussion}

$\nabla$

\section{Methodik der Studie - Limitationen}

Einzel- und Fokusgruppeninterviews mit Experten erwiesen sich für die vorliegende Studie als geeignete Erhebungsmethode zur Beschreibung des Angebots bzw. zur Erfassung von Haltungen und Präferenzen zur „Psychosomatischen Sprechstunde im Betrieb“. Bei der Rekrutierung konnte eine strukturelle Variation in Bezug auf Betriebsgröße, Branche, Art der betriebsärztlichen Betreuung und Infrastruktur (Großstadt bis ländliche Gebiete) erreicht werden. Die Bewertungen der PSIB zeugen von einer hohen Zufriedenheit, grundlegende Kritik wurde kaum geäußert. Auch zeigten die interviewten Betriebsärzte ein hohes Interesse an ihrer eigenen Einbindung in den Prozess der Psychosomatischen Sprechstunde. Beide Aspekte könnten Hinweise auf einen möglichen Bias geben: Denkbar ist, dass bei den Befragten „mit Erfahrung“ Personen interviewt wurden, die das Angebot initiiert haben und deshalb Kritik und Zweifel seltener thematisierten. Gleichzeitig könnten durch eine Rekrutierung der Betriebsärzte „ohne Erfahrung“ über das Netzwerk des Instituts besonders engagierte Betriebsärzte erreicht worden sein. Möglicherweise hätten andere Betriebsärzte ein geringeres Interesse an einem konsiliarisch ausgestalteten Angebot der „Psychosomatischen Sprechstunde im Betrieb“ gezeigt oder weniger Befürchtungen im Hinblick auf das Entstehen einer Parallelstruktur geäußert.

Die Rekrutierung von Interviewpersonen war nur zeitlich begrenzt möglich (Projektlaufzeit), sodass eine „Sättigung der Stu- dienpopulation“ [16] nicht garantiert ist und mit weiteren Experten vielleicht neue Aspekte in das Datenmaterial Eingang gefunden hätten. Da sich im Datenmaterial immer wiederkehrende Themen herauskristallisierten, ist allerdings davon auszugehen, dass die Datenbasis zentrale Themen repräsentiert.

\section{Diskussion der Ergebnisse}

Der hier wiedergegebene Ausschnitt der Studie befasste sich mit der Gestaltung des neuen Versorgungsangebots PSIB und den damit verbundenen Aspekten Information über das Angebot, Zugang bzw. Zuweisung, Ort und Behandlungsdauer. Über diese Gestaltungsaspekte lassen sich ein eher „restriktives“ und ein eher „liberales“ Modell unterscheiden, wobei vor allem die beiden Aspekte „Information“ und „Zugang“ eng verbunden sind mit dem Grad der Einbindung des Betriebsarztes. Das „restriktive“ Modell erfüllt hierbei am ehesten Charakteristika, die für die Konsiliarpsychosomatik im stationären Bereich [10] beschrieben wurden: Der Betriebsarzt ist dabei derjenige, der ausgewählte Beschäftigte über die Psychosomatische Sprechstunde informiert. Entsprechend stellt allein der Betriebsarzt die Indikation und weist der Psychosomatischen Sprechstunde zu und wird so im „restriktiven“ Modell zu einem zentralen Akteur einer konsiliarisch angelegten PSIB. Im „liberalen Modell“ nimmt der Betriebsarzt keine zentrale Rolle ein. Hier werden alle Beschäftigten unabhängig von einer konkreten Bedarfssituation über das Angebot informiert und können bei unterschiedlichen Zuweisern ihren Bedarf zur Psychosomatischen Sprechstunde äußern. Der Betriebsarzt wird lediglich informiert. Im vorliegenden Material ist ein in Bezug auf die Aspekte „Information“ und „Zugang“ „liberales“ Modell ganz ohne Einbeziehung des Betriebsarztes nicht abgebildet - möglicherweise aufgrund der Zusammensetzung der Stichprobe.

Die Psychosomatische Sprechstunde kann in betriebseigenen Räumlichkeiten oder betriebsextern stattfinden. Die Anonymität wird im ersten Fall durch eine Ansiedlung in betriebsärztlichen Räumlichkeiten und im anderen durch die externe Örtlichkeit gefördert.

Der Gestaltungsaspekt „Umfang der Behandlung“ ist eng verbunden mit einer Kosten-Nutzen-Abwägung im Betrieb. Umfasst die „Psychosomatische Sprechstunde“ nur ein Erstgespräch, soll dieses bewirken, dass der Beschäftigte gegebenenfalls mit Unterstützung des Betriebsarztes oder weiterer betrieblicher Angebote Lösungen für seine Problematik findet oder dass er ein Verständnis für seine psychosomatischen Zusammenhänge entwickelt und motiviert ist, Angebote der Regelversorgung wahrzunehmen. Im anderen Konzept können Beschäftigte bis zu 5 Termine der „Psychosomatischen Sprechstunde“ in Anspruch

Tab. 4 Charakteristika der Psychosomatischen Sprechstunde in den 5 betrachteten Betrieben \# in Bezug auf die Gestaltungsaspekte „Information“, „Zugang“, „Ort der Sprechstunde“ und „Umfang der Behandlung“. (" Betriebe, deren Betriebsärzte an der Fokusgruppe FG_AMM-01 teilnahmen)

\begin{tabular}{|c|c|c|c|c|c|c|}
\hline \multirow{2}{*}{ Betrieb } & \multicolumn{2}{|c|}{ Information } & \multicolumn{2}{|c|}{ Zugang } & \multicolumn{2}{|c|}{ Umfang der Behandung } \\
\hline & $\begin{array}{l}\text { gezielt in der (betriebsärztli- } \\
\text { chen) Sprechstunde }\end{array}$ & an alle Beschäftigte & über Betriebsarzt & mehrere Zuweisende & 1 Termin & 5 Termine \\
\hline A & & $x$ & $x$ & & $x$ & \\
\hline B & $x^{*}$ & & & $x$ & $x$ & \\
\hline C & $x$ & & $x$ & & & $\mathrm{x}^{* *}$ \\
\hline D & & $x$ & & $x$ & & $\mathrm{x}$ \\
\hline $\mathbf{E}$ & $x$ & & $x$ & & & $x$ \\
\hline
\end{tabular}


nehmen - womit gegebenenfalls bereits eine therapeutische Wirkung erzielt wird.

Die Umsetzung der „Psychosomatischen Sprechstunde im Betrieb“ erfolgt somit aktuell heterogen; die verschiedenen Gestaltungsaspekte werden in der betrieblichen Praxis entsprechend der jeweiligen Erfordernisse unterschiedlich kombiniert ( $\bullet$ Tab. 4). Diese Vielfalt bewertete das Fachpublikum im Validierungsworkshop als positiv, da so ein bedarfsgerechter Zuschnitt des Angebots an die betrieb(särzt)lichen Gegebenheiten und die Möglichkeiten des Konsiliars erfolgen könne. Ausgehend von den vorliegenden Erkenntnissen sollten die genannten Aspekte bei Neuimplementierungen des Angebots und dessen Evaluation [17] bedacht werden. Empfehlungen für ein bestimmtes Modell oder einzelne Komponenten können derzeit nicht abgegeben werden, da bislang quantitative Daten zur Effektivität der einzelnen Modelle fehlen.

Zu bedenken ist, dass mit der „Psychosomatischen Sprechstunde im Betrieb“ ein Arbeitgeber-finanziertes Angebot entsteht, das aufgrund der Adressierung erkrankter/symptomatischer Beschäftigter letztlich der Regelversorgung zuzuordnen ist. Sollte die aktuell laufende erste Evaluation [17] dessen Effektivität belegen, könnte die Kostenübernahme durch die gesetzliche Krankenversicherung erwogen werden. Spätestens dann sollten ausgehend von den hier vorgelegten empirischen Daten - Rahmenbedingungen für die „Psychosomatische Sprechstunde im Betrieb“ und gegebenenfalls weitere therapeutische Angebote im Betrieb (z. B Psychosomatische Kurzzeittherapie [18]) entwickelt und fixiert werden. Alternativ könnte die Versorgung von Beschäftigten außerhalb des Betriebs beschleunigt werden, wie dies durch Anreizsysteme wie Sondervergütungen für niedergelassene Psychotherapeuten [19] oder Modelle der Integrierten Versorgung versucht wird. In jedem Fall sollten in die Entwicklung betriebsnah ausgerichteter Angebote der Krankenversorgung alle Beteiligten, d.h. Betriebsärzte, betriebliche Akteure und psychosomatische/psychotherapeutische Konsiliare gleichermaßen eingebunden werden.

\section{Danksagung}

Die Autorinnen danken allen Interviewpartnerinnen und Interviewpartnern für die Mitwirkung an der Studie. Frau Dr. Eva Rothermund und Herrn Prof. Dr. Harald Gündel (Universitätsklinik für Psychosomatische Medizin und Psychotherapie, Ulm), Herrn PD Dr. Michael Hölzer (Sonnenbergklinik, Stuttgart) und Frau Dr. Dorothea Mayer (Werksärztlicher Dienst Sindelfingen, Daimler AG) danken wir für den fruchtbaren Gedankenaustausch zum Thema „Psychosomatische Sprechstunde im Betrieb“. Die Studie wurde gefördert durch das Ministerium für Arbeit und Sozialordnung, Familie, Frauen und Senioren Baden-Württemberg. Die Arbeit des Instituts für Arbeitsmedizin, Sozialmedizin und Versorgungsforschung Tübingen wird finanziell unterstützt durch den Verband der Metall- und Elektroindustrie BadenWürttemberg e.V. (Südwestmetall).

Interessenkonflikt: Die Autoren geben an, dass kein Interessenkonflikt besteht.

\section{Literatur}

1 DAK-Gesundheit. DAK-Gesundheitsreport 2013. Im Internet: http:// www.presse.dak.de/ps.nsf/Show/998583CFE0F4B967C1257B18004 DA198/\$File/Gesundheitsreport_2013_Druckfassung \%2015.2.2013 PubMed pdf; Stand: 13.06.2013

2 Deutsches Ärzteblatt, Ressort Politik. Psychische Erkrankungen Hauptursache für Frühverrentung. 2013. Im Internet: http://www.aerz teblatt.de/nachrichten/52875/Psychische-Erkrankungen-Hauptursa che-fuer-Fruehverrentung Stand: 22.05.2013 (Last accessed on 22 May 2013)

3 Klein S, Appelt HJ., Hrsg. Praxishandbuch betriebliche Sozialarbeit. Konzepte und Methoden in Theorie und Praxis. Kröning: Asanger; 2010

4 Deutsche Hauptstelle für Suchtfragen (DHS). Qualitätsstandards in der betrieblichen Suchtprävention und Suchthilfe der Deutschen Hauptstelle für Suchtfragen (DHS). Ein Leitfaden für die Praxis. 2011. Im Internet: http://www.dhs.de/arbeitsfelder/arbeitsplatz.html Stand: 19.05.2013

5 Moos S, Wittich A. Coaching für Ärztinnen in der Facharztausbildung. In: Organisationsberatung Supervision Coaching 2012; $19: 227-235$

6 Bundesministerium für Arbeit und Soziales. Arbeitsmedizinische Empfehlung (AME) „Psychische Gesundheit im Betrieb“. Eigenverlag 2008

7 Schulz H, Barghaan D, Harfst T et al. Psychotherapeutische Versorgung. Gesundheitsberichterstattung des Bundes. Themenheft 41. Eigenverlag 2008; 1-47

8 Kruse J, Herzog W. Zur psychosomatischen/psychotherapeutischen Versorgung in der kassenärztlichen Versorgung in Deutschland - Formen der Versorgung und ihre Effizienz. Zwischenbericht. Gutachten im Auftrag der Kassenärztlichen Bundesvereinigung. Heidelberg, Gießen: Eigenverlag; 2012

9 Mayer D, Schmidt H, Hölzer M. „Psychosomatische Sprechstunde“ und „Psychosomatische Grundversorgung in der Arbeitsmedizin“. Erfahrungen aus der Automobil-Industrie. Arbeitsmed Sozialmed Umweltmed 2010; 45: 593-597

10 Söllner W, Stein B. Konsiliar- und Liasonpsychosomatik. In: Uexküll Tvon, Hrsg. Psychosomatische Medizin. Theoretische Modelle und klinische Praxis. München: Elsevier, Urban \& Fischer; 2011; 543-552 PubMed

11 Craig P, Dieppe P, Macintyre S et al. Medical Research Council Guidance: developing and evaluating complex interventions. The new Medical Research Council guidance. In: BMJ 2008; 337: 979-983

12 Pzyborski A, Riegler J.. Gruppendiskussion und Fokusgruppe. In: Mey G, Mruck K, Hrsg. Handbuch der qualitativen Forschung in der Psychologie. Wiesbaden: VS Verlag; 2010; 436-448 PubMed

13 Meuser M, Nagel U. Das Experteninterview - konzeptionelle Grundlagen und methodischen Anlage. In: Pickel S, Pickel G, Lauth HJ, Jahn D, Hrsg. Methoden der vergleichenden Politik- und Sozialwissenschaft. Neue Entwicklungen und Anwendungen. Wiesbaden: VS Verlag; 2009; 465-479

14 Kleining G. Umriss zu einer Methodologie Qualitativer Sozialforschung. Kölner Zeitschrift für Soziologie und Sozialpsychologie 1982; 34: $224-253$

15 Mayring P. Qualitative Inhaltsanalyse. Grundlagen und Techniken. Weinheim. Beltz; Verlag: 2003

16 Glaser BG, Strauss AL. Groundedd Theory. Grundlagen qualitativer Forschung. Bern: Huber; 1998

17 Rothermund E, Kilian R, Hölzer $M$ et al. "Psychosomatic consultation in the workplace" - a new model of care at the interface of companysupported mental health care and consultation-liaison psychosomatics. Design of a mixed methods implementation study. BMC Public Health 2012; 12: 780

18 Hölzer M. Psychische Gesundheit im Betrieb. Die psychosomatische Kurzzeittherapie. Psychotherapie im Dialog 2012; 13: 52-56

19 Bühring $P$. Versorgung psychisch Kranker in Baden-Württemberg. Erstgespräch in drei Tagen. Dtsch Arztebl 2011; 108 